states, one to a transition from state 0 to state 1 , the other from state 1 to state 0 . Both these states may be expected to occur under ordinary conditions when the moment of inertia of the molecule is large, as in the case of carbon dioxide.

These modified lines show that the model of carbon dioxide is a linear one as considered by Eucken (Zeits. f. Physik, 37, 714 ; 1926), who has calculated the frequency corresponding to the vibration along the direction joining the $\mathrm{C}$ - and $\mathrm{O}$-atoms to be of that of an anharmonic oscillator having a frequency corresponding to $7 \cdot 86 \mu$-this frequency being optically inactive in the case of absorption. In the case of scattering, this frequency will evidently be the one which will be the most prominent. Of the other two fundamentals at $4.25 \mu$ and $14.87 \mu$, one may be optically inactive in the case of scattering, and the intensity contribution of the other may be so feeble that it would require a very large exposure to show its presence. It may here be pointed out that the pair of bands at $2.7 \mu$, which has been considered as a fundamental by Schaefer and Phillipps, has been shown by Eucken as due to combination frequencies, and one is not justified in considering it as a fundamental due to its weak intensity. That the carbon dioxide molecule has a linear model is also shown by the absence of a permanent dipole moment and from the ordinary scattering data.

Thus the observations of Rasetti are a very clear proof not only of the Raman effect, but also of the linear model of carbon dioxide. It is also expected that in the other triatomic molecules where the dielectric constant data show the absence of a permanent dipole moment due to linear structure with symmetrical disposition of the two atoms on each side of the central one, the Raman effect would be shown evidently with less exposure for the optically inactive fundamental frequency in absorption.

P. N. Ghosh.

P. C. Mahanti.

Applied Physics Laboratory,

University College of Science,

92 Upper Circular Road, Calcutta.

\section{Further Investigation on Incoherent Scattering in Gases.}

IN letters to NATURE, and in two papers published in the Proc. Nat. Acad. Sci. of America, I have reported some results obtained on the Raman effect in gases. I want to report now about some new experiments, which will be more completely described in the Physical Review.

I have observed, for the first time, so far as I know, a purely electronic transition in the scattering process. The molecule NO has a normal ${ }^{2} P$ state, the separation of the doublet being about $124 \mathrm{~cm}^{-1}$; the two levels arise from the two opposite orientations of the electronic spin relatively to $i_{l}$, that is, to the nuclear axis. I have found this transition in the Raman spectrum excited by $\lambda 2536$.

I have built a new apparatus in order to work with gases at 10 atmospheres pressure, with ultra-violet excitation. In this way I have obtained beautiful Raman spectra of hydrogen. They show eight lines corresponding to purely rotational transitions, and also well-developed vibrational-rotational Raman bands, of which so far only unresolved $Q$-form branches had been recorded. I have measured now three lines of the $Q$-form branch, four of the $R R$-form branch, and one of the $P P$-form branch for the transition from the zero to the first vibrational level.

From this, not only the moment of inertia and the vibration frequency of the $\mathrm{H}_{2}$ molecule can be calculated with high accuracy, but also the coupling between rotation and vibration.

Gaseous hydrocarbons at ten atmospheres give strong Raman lines in a few hours' exposure. Systematic work is being extended in this direction.

\section{F. RASETti}

\section{California Institute of Technology,} Pasadena, June 1.

\section{Statistics in Biological Research.}

IN the review of Dr. R. A. Fisher's " Statistical Methods for Research Workers " in NATURE of June 8, the point is made that a careless reader may get the impression that the various methods outlined therein will give exact results when applied to the ordinary small 'sample', although we have, in general, no proof, or even expectation, that the sample is drawn from a 'normal' population, to which alone the tables can be exactly applied. That this is so is clear from the fact that an American writer has stated that the English school of statisticians claims to have produced tables which may be used for samples, however small, drawn from any conceivable population; but of course Dr. Fisher would be the first to cry out against the foolishness of making any such claim.

That such a misconception should arise is perhaps not unnatural when a mathematician is trying to explain what he has been doing to those who lack the mathematical outlook, but this would presumably not apply to the reviewer; yet by his use of the word "admit, when doubtless he meant "stress" ("It would seem wiser ... to admit the incompleteness of theory...") he runs the risk of seeming to support a misstatement which Dr. Fisher may well resent.

The question of the applicability of normal theory to non-normal material is, however, of considerable importance and merits attention both from the mathematician and from those of us whose province it is to apply the results of his labours to practical work. Personally, I have always believed, without perhaps any very definite grounds for this belief, that in point of fact 'Student's' distribution will be found to be very little affected by the sort of small departures from normality which obtain in most biological and experimental work, and recent work on small samples confirms me in this belief. We should all of us, however, be grateful to Dr. Fisher if he would show us elsewhere on theoretical grounds what sort of modification of his tables we require to make when the samples with which we are working are drawn from populations which are neither symmetrical nor mesokurtic.

The Galton Laboratory, University College, London.

\section{The Long Period Variations of UZ Persei.}

In Harvard College Observatory Bulletin, No. 867 , Feb. 1, 1929, there is a note by B. P. Gerasimovič on "UZ Persei, a Variable of Unusually Long Period". The author found that the brightness of this variable star has two oscillations - short and long - with periods of about 90 and 907 days respectively. It is known that such oscillations are typical for $\mu$ Cephei.

In a short note (Astronomical Bulletin, Mirowedenije, Nr. 22, 1928) I showed from 3785 observations that $\mu$ Cephei has three oscillations. The periods of these oscillations are 90 days, 600 days and 13 years. The most interesting is the last oscillation, on account 